\title{
Parametric adjustment in saccadic eye movements'
}

During a change-of-fixation eye movement, the target toward which $S$ was shifting his gaze was displaced $1^{\circ}$ toward the original point of fixation so that the eye made an overshoot with respect to the new target position. When this was repeated several times in succession, the eye movement control system made an adjustment such that the overshoot gradually diminished. The end-result of this "parametric adjustment" was that a visual target $10^{\circ}$ from the fovea elicited an eye movement of only $9.1^{\circ}$.

One of the most remarkable properties of the human eye-movement control system is its ability to execute rapid and accurate changes of fixation. Consider, for example, the situation in which $S$ is instructed to fixate a target which (prior to the change of fixation) is imaged on his retina $10^{\circ}$ from the fovea. After the reaction time which precedes any voluntary act $(.15-.20 \mathrm{sec})$, the eye movement itself takes only about .04 sec to achieve $95 \%$ accuracy.

This performance is all the more remarkable when one considers that the time involved $(.04 \mathrm{sec})$ is far too short for the operation of any exteroceptive feedback loop which might guide the eye to the target in successive approximations (Dodge \& Cline, 1901, p. 149); that the visual aculty $10^{\circ}$ from the fovea is only $1 / 5$ of what it is at the fovea (Ludvigh, 1941); and that the eye is lacking in articular sensitivity (Merton, 1964). Furthermore, because of variations in such parameters as fatigue, starting position of the eye movement, and resting tonus of the muscles, a given pattern of innervation may produce different amounts of ocular rotation at different times.

How can we account for the ability of the control system to operate as well as it does under these adverse conditions? One possible explanation is that the system is self-correcting: when it makes an error (overshoot or undershoot), it adjusts its own parameters so as to reduce the probability that the error will recur. For example, if a change-of-fixation eye movement falls short of the target, so that one or more secondary corrective movements are required to achieve accurate foveal fixation, then (according to this hypothesis) the characteristics of the control system will be altered so that, on the next try, a visual target in the same extrafoveal position will elicit a larger eye movement. The adjustment may take place in some neural center where efferent impulses to the extrinsic eye muscles are modified, or there may be a change in the resting tonus of the muscles, or some other parameter may be affected.
An adjustment of this sort, which alters the relationship between voluntary direction of gaze and the resulting ocular position, will be referred to here as a "parametric adjustment." This term was used by E. Ludvigh (1952, p. 445) to designate an adjustment made in response to afferent signals from muscle spindle receptors in the extrinsic eye muscles. The present usage extends Ludvigh's concept to include adjustments made in response to errors in fixation.

The purpose of the present experiment was to find out whether or not the human oculomotor control system is capable of making a parametric adjustment in response to its own fixation error.

\section{METHOD}

Figure 1 shows the apparatus in plan view. A bite

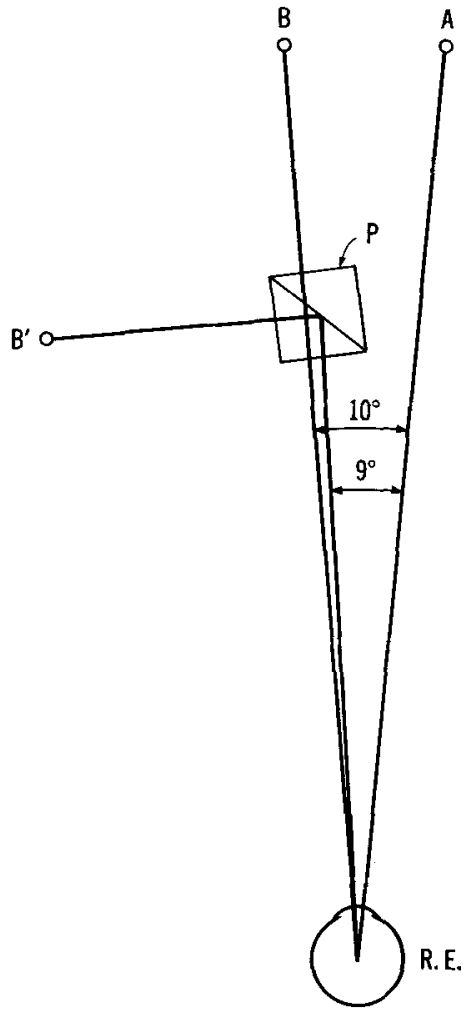

Fig. 1. Schematic drawing of the apparatus in plan view. $A, B$, and $B^{\prime}$ are stimulus lights; $P$ is a semi-reflecting prism; RE is $S^{\prime}$ s right eye. The S's task was simply to shift his gaze from $A$ to $B$. During the eye movement, $B$ was switched off and $B$ ' (indistinguishable from B) was switched on, thus inducing an overshoot with respect to $\mathbf{B}^{\prime}$. 


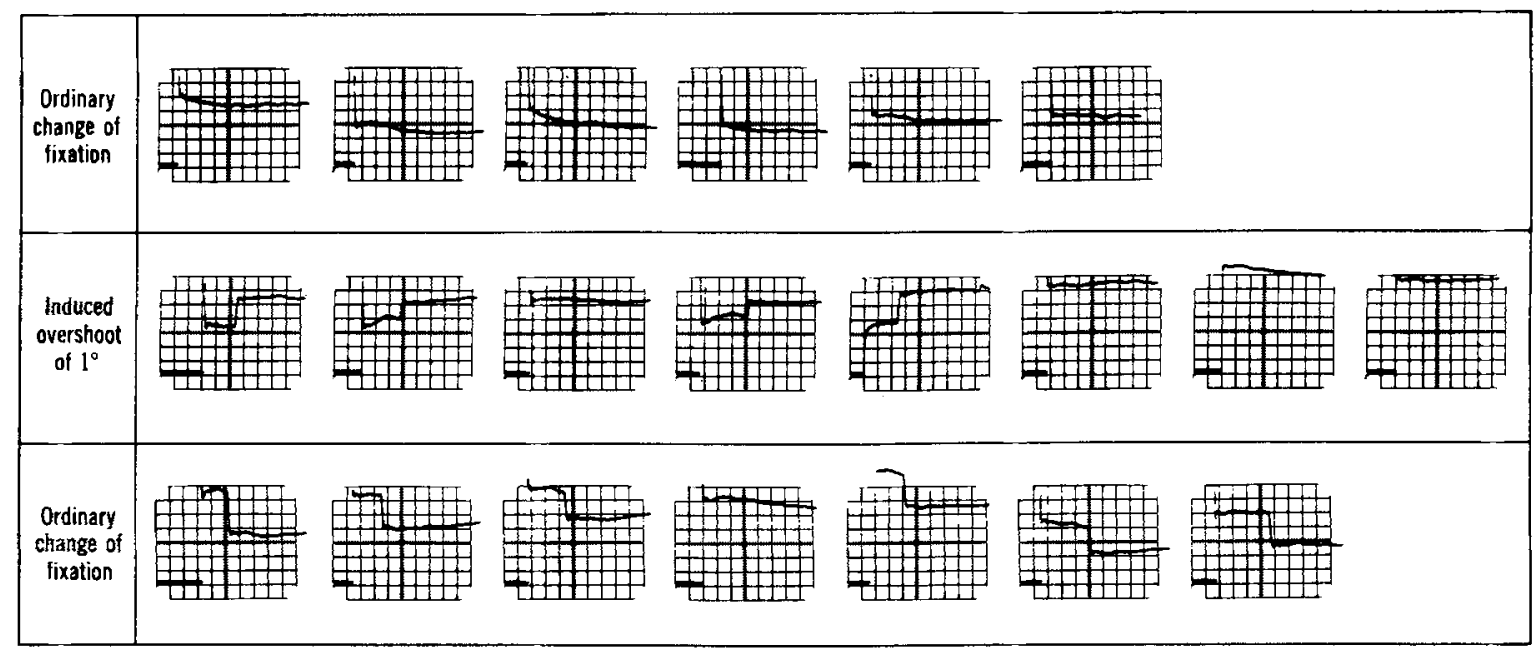

Fig. 2. Oscilloscope traces of 21 consecutive A-to-B eye movements (right eye only). The interval between successive eye movements was 3-4 sec. The sweep was triggered simultaneously with the buzzer which was S's signal to shift his gaze. Sweep time was $0.1 \mathrm{sec}$ per horizontal division, direction left to right. Vertical calibration $30 \mathrm{~min}$ arc per vertical division. A movement downward on the record represents an ocular rotation toward S's left. Only the last $2-5^{\circ}$ of the $10^{\circ}$ eye movement appears on the scope. The horizontal line at the bottom of the record is the stimulus trace: the discontinuity in it represents the moment of $\mathbf{B}-\mathbf{B}^{\prime}$ switching. The first six eye movements were made with no switching, the next eight with induced overshoot, and the last seven with no switching.

plate bearing S's dental impression fixed his head in position, and his left eye was occluded. The viewing distance was $33 \mathrm{~cm}$.

The light $A$ was $2 \mathrm{~mm}$ in diameter. The light $B$ was $2 \mathrm{~mm}$ wide and $30 \mathrm{~mm}$ high. Each light had cross-hairs in its center, and $S$ was instructed to fixate the cross-hairs on each light in turn.

The experiment started with $S$ fixating the light $A$, while the light $B\left(10^{\circ}\right.$ to the left of A) was visible in the periphery of his field of view. On signal (a buzzer), S shifted his gaze to $B$. Approximately 1.5 sec after the buzzer had sounded, $S$ again fixated $A$ and walted for the buzzer to sound.

While flxating $A$ and walting for the buzzer, $S$ was instructed to blink several times. About $1 \mathrm{sec}$ before the buzzer sounded, he was instructed to stop blinking and to refrain from blinking until the $A-$ to-B movement had been completed.

Each A-to-B eye movement was monitored and recorded by means of a detection system based on the principle described by Torok, Guilleman, and Barnothy (1951), by Smith and Warter (1960), and by Stark, Vossius, and Young (1962). The principle utilizes the difference in reflectivity between iris (dark) and sclera (white). In the present experiment, the optical element was a spherical reflector $30 \mathrm{~cm}$ in diameter, positioned $50 \mathrm{~cm}$ from S's eye with its optical axis $37^{\circ}$ to the right of S's straight-ahead line of sight. On the same axis, $25 \mathrm{~cm}$ from S's eye, was an infrared-sensitive semiconductor photodiode (Texas Instruments LS-400), positioned so that the image formed by the spherical reflector was incident on the light- sensitive surface of the diode. The output of this system was a voltage proportional to rotational position of the eye in a horizontal meridian.

The light $B$ was seen through the semi-reflecting prism, and the light $B^{\prime}$, indistinguishable from $B$, was seen via the prism. These two lights were never on simultaneously; and the one that was off was not visible to $S$. As seen by $S, B^{\prime}$ appeared $1^{\circ}$ to the right of $B$. The use of a semi-reflecting prism made it possible to set up $B-B^{\prime}$ separations less than the width of either light; but preliminary investigations showed that the experiment would work with separations at least as great as $6 \mathrm{~mm}(10)$.

\section{RESULTS}

Figure 2 shows the results obtained with one $S$ in a sequence of 21 A-to-B eye movements. The $S$ remained on the bite plate throughout the sequence. The first six eye movements were ordinary changes of fixation, with no B-B' switching; in the next eight eye movements, the $B-B^{\prime}$ switching occurred at the point indicated on the stimulus trace; and the last seven eye movements were ordinary changes of fixation with no switching.

For numerical analysis of these records, it was necessary to allow for the fact that the voltage output of the detector does not provide a reliable measure of absolute position of the eye over the entire sequence. One possible source of error is a change in head position during the sequence (a sequence lasts 1-2 $\mathrm{min}$ ). Another possible source of error in records of this type is that the drying of the surface of the eye be- 
tween blinks may change its reflectivity, thus changing the output of the detector (Krauskopf, Graf, \& Gaarder, 1966, p. 75).

The assumption was therefore made that the eye achieved precise foveal fixation $( \pm 5$ min of arc; see Barlow, 1952) within 1 sec after the buzzer signali.e., by the end of one oscllloscope sweep. On this assumption, the terminal value of each eye movement record represents foveal fixation of the target, and the difference between that value and the terminal value of the initial saccade represents the amount of overshoot or undershoot in the initial saccade.

Because of the indeterminacy of the end-point of a saccade, the eye position $0.1 \mathrm{sec}$ after the moment of $B-B^{\prime}$ switching was taken as the terminal locus of the initial saccade. The moment of switching was available as a reference mark on the stimulus trace even when the lights were not actually switched.

Figure 3 shows the mean fixation-error data for nine sequences similar to that in Fig. 2. Included in these nine sequences are five for the $S$ of Fig. 1 , three for a second $S$, and one for a third $S$.

\section{INTERPRETATION OF RESULTS}

The first three eye movements in Fig. 3 show a tendency toward undershoot in ordinary change of fixation, which agrees with the findings of previous investigators for saccades of this order of magnitude (Clark, 1936; Hyde, 1959). The next two eye movements show the overshoot induced by the B-B' switching; and the next three after that show the presence of a parametric adjustment such that a visual target at $10^{\circ}$ elicits an eye movement of only a little over $9^{\circ}$. On the return to ordinary change of fixation, the $10^{\circ}$ target again elicits an eye movement of approximately $9^{\circ}$ (mean value $9.07^{\circ}$ ).

During the last four eye movements, error feed-

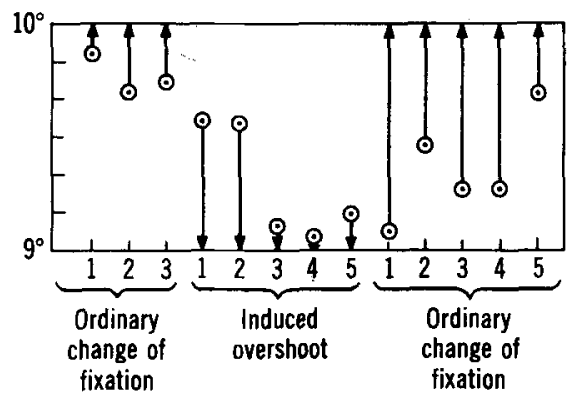

Fig. 3. Mean fixation-error data for nine sequences similar to that shown in Fig. 2. For each eye movement, the plotted point shows the terminal locus of the initial saccade, and the arrow shows the direction and magnitude of the corrective eye movements which were necessary to bring the eye onto the target. Only the last three eye movements in the Iirst "ordinary change of fixation" group are shown. In the "induced overshoot" group, only the first three and the last two eye movements are shown. This was done in order to make it possible to combine sequences in which the number of eye movements with induced overshoot varied between five and nine. back signals cause a new parametric adjustment which returns the system to the operating state which it had at the start of the sequence. This second parametric adjustment appears slower than the first, perhaps because it works against, rather than with, the tendency toward undershoot.

\section{DISCUSSION}

It is clear from these results that the human oculomotor system can and does respond to its own error by making a parametric adjustment which tends to decrease the likelihood that the error will recur.

It might be suggested that $S$ made a conscious correction rather than a parametric adjustment. However, $S$ was not usually aware of the B-B' switching at all; and, when he was aware of it, he found himself looking at the light in its new position before he was aware that it had changed position.

In addition to the type of parametric adjustment reported here, there is another type which occurs in vergence and duction movements (Ludvigh, 1952) and in adaptation to prismatic displacement (McLaughlin, Rifkin, \& Webster, 1966; McLaughlin \& Webster, 1967). Both types involve an alteration in the relationship between voluntary direction of gaze and eye position. The difference between the two is that one affects the static position of the eye while the other affects only change of fixation. In prism adaptation, for example, the effect of a parametric adjustment is to add a constant error to the judgment of the direction of gaze: $S$ may believe that he is looking straight ahead when in fact his eyes are directed off to the side (McLaughlin \& Webster, 1967). By contrast, the type of adjustment shown in Figs. 2 and 3 of the present paper does not affect the momentary position of the eye or the judgment of the direction of gaze. There is no evidence for the presence of the adjustment until $S$ changes fixation from one object to another, at which time an abnormally large overshoot or undershoot will be observed.

\section{References}

Barlow, H. B. Eye movements during fixation. J. Physiol., 1952, $116,290-306$.

Clark, B. The effect of interfixation distance on fixation movements. J. exp. Psychol., 1936, 19, 505-512.

Dodge, R., \& Cline, $\mathbf{T}$. The angle velocity of eye movements. Psychol. Rev., 1901, 8, 145- 157.

Hyde, J. Some characteristics of voluntary human ocular movements in the horizontal plane. Amer. J. Ophthal., 1959, 48, 85-94.

Krauskopf, J., Graf, V., \& Gaarder, K. Lack of inhibition during voluntary saccades. Amer. J. Psychol., 1966, 79, 73-81.

Ludvigh. E. Extrafoveal visual acuity as measured with Snellen test-letters. Amer. J. Ophthal., 1941, 24, 303-310.

Ludvigh, E. Control of ocular movements and visual interpretation of the environment. Atch. Ophthal., 1952, 48, 442-448.

McLaughlin, S. C., Rifkin, K. I., \& Webster, R. G. Oculomotor adaptation to wedge prisms with no part of the body seen. Percept \& Psychophys., 1966, 1, 452-453.

McLaughlin, S. C., \& Webster, R. G. Changes in straight-ahead eye position during adaptation to wedge prisms. Percept. \& Psychophys., 1967, 2, 36-44. 
Merton, P. A. Absence of conscious position sense in the human eye. In M. B. Bender (Ed.), The oculomotor system. New York: Harper \& Row, 1964. Ch. 14.

Smith, W. M., \& Warter, P. J. Eye movement and stimulus movement; new photoelectric electromechanical system for recording and measuring tracking motions of the eye. J. Opt. Soc. Amez. $1960,50,245-250$.

Stark, L., Vossius, G., \& Young, L. R. Predictive control of eye tracking movements. I.R.E. Trans, on Human Factors in Electronics, $1962,3,52-56$.
Torok, N., Guillemin, V., \& Bamothy, J. Photoelectric nystagmography. Ann. Otol. Rhinol., \& Laryngol., 1951, 60, 917-926.

\section{Note}

1. The research described here was supported in part by contract MD-2714 with the U. S. Army Medical Research and Development Command, and in part by Research Grant GB-4196 from the National Science Foundation.

(Accepted for publication April 10, 1967.) 